\title{
Intensive care unit discharge to the ward with a tracheostomy cannula as a risk factor for mortality: A prospective, multicenter propensity analysis
}

Rafael Fernandez, MD; Ana-Isabel Tizon, MD; Javier Gonzalez, MD; Pablo Monedero, MD; Manuela Garcia-Sanchez, MD; Ma-Victoria de-la-Torre, MD; Pedro Ibañez, MD; Fernando Frutos, MD; Frutos del-Nogal, MD; Ma-Jesus Gomez, MD; Alfredo Marcos, MD; Gonzalo Hernández, MD; Sabadell Score Group.

From the Intensive Care Unit (RF), Hospital Sant Joan de Deu- Fundacio Althaia, Manresa; Universitat Internacional de Catalunya (RF), Catalunya; Intensive Care Unit (AIT), Hospital Xeral Cies, Vigo; Intensive Care Unit (JG), Hospital de Salamanca, Salamanca; Intensive Care Unit (PM), Clinica Universidad de Navarra, Navarra; Intensive Care Unit (MGS), Hospital Universitario Virgen Macarena, Sevilla; Intensive Care Unit (MVDLT), Hospital Virgen de la Victoria, Malaga; Intensive Care Unit (PI), Hospital Son Llatzer, Mallorca; Intensive Care Unit (FF), Hospital Universitario de Getafe, Getafe; CIBER Enfermedades Respiratorias (FF), Bunyola; Intensive Care Unit (FDN), Hospital Severo Ochoa, Leganes; Intensive Care Unit (MJG), Hospital General Universitario Reina Sofia, Murcia; Intensive Care Unit (AM), Hospital Virgen de la Concha, Zamora; and Intensive Care Unit (GH), Hospital Infanta Sofia, San Sebastian de los Reyes, Spain.

The authors have not disclosed any potential conflicts of interest.

For information regarding this article, E-mail: rfernandez@althaia.cat

Objective. To analyze the impact of decannulation before intensive care unit discharge on ward survival in nonexperimental conditions.

Design. Prospective, observational survey.

Setting. Thirty-one intensive care units throughout Spain.

Patients. All patients admitted from March 1, 2008 to May 31, 2008.

Interventions. None.

Measurements and Main Results. At intensive care unit discharge, we recorded demographic variables, severity score, and intensive care unit treatments, with special attention to tracheostomy. After intensive care unit discharge, we recorded intensive care unit readmission and hospital survival. Statistics: Multivariate analyses for ward mortality, with Cox proportional hazard ratio adjusted for propensity score for intensive care unit decannulation. We included 4,132 patients, 1,996 of whom needed mechanical ventilation. Of these, 260 (13\%) were tracheostomized and 59 (23\%) died in the intensive care unit. Of the 201 intensive care unit tracheostomized survivors, 60 were decannulated in the intensive care unit and 141 were discharged to the ward with cannulae in place. Variables associated with intensive care unit decannulation (nonneurologic disease [85\% vs. 64\%], vasoactive drugs [90\% vs. $76 \%$ ], parenteral nutrition [55\% vs. $33 \%$ ], acute renal failure [37\% vs. $23 \%$ ], and good prognosis at intensive care unit discharge [40\% vs. 18\%]) were included in a propensity score model for decannulation. Crude ward mortality was similar in decannulated and nondecannulated patients (22\% vs. 23\%); however, after adjustment for the propensity score and Sabadell Score, the presence of a tracheostomy cannula was not associated with any survival disadvantage with an odds ratio of $0.6[0.3-1.2](p=.1)$.

Conclusion. In our multicenter setting, intensive care unit discharge before decannulation is not a risk factor. (Crit Care Med 2011; 39:2240-2245)

Key words: hospital survival; mechanical ventilation; outcome research; propensity score; tracheostomy. 


\section{INTRODUCTION}

Between $10 \%$ and $40 \%$ of patients with prolonged mechanical ventilation (MV) undergo tracheostomy either to improve comfort during prolonged MV or to shorten weaning (1-3). Whether tracheostomy improves the outcome of MV patients is controversial (4-6). Some studies report that tracheostomy expedites discharge to the ward, allowing better intensive care unit (ICU) resource allocation (7). However, some studies report higher ward mortality in cannulated patients $(8,9)$; thus, decannulation before ICU discharge is advocated. Nevertheless, extending the ICU stay to enable decannulation is expensive and occupies scarce ICU beds. A recent study reported that surveillance of tracheostomized patients in the ward by a dedicated team shortened the length of stay and increased survival (10).

One observational study found that decannulation before ICU discharge decreased the risk of mortality in the ward in patients with poor short-term or long-term prognosis but had no effect on mortality in patients with good prognosis or in those expected to die in the hospital (11).

A randomized clinical trial would be the best approach to definitively determine the possible clinical benefit of decannulation before ICU discharge, but the need to prolong ICU stays makes such trials unfeasible given the shortage of available ICU beds. In recent years, propensity scores have become common to adjust for confounding factors in observational studies (6). The propensity score is a predicted probability resulting from a model. Its main purpose is to control multiple confounders simultaneously and to account for treatment indication bias, and it is useful for maximizing the balance between the treatment groups. Clec'h et al (6) suggested that decannulation before ICU discharge is associated with better ward survival, but their propensity analysis was focused on the factors associated with tracheostomy, without a deep analysis of factors associated with decannulation.

Therefore, we hypothesized that a sensitive observational analysis of the out-come of ICU tracheostomized patients may add important information about the possible benefits of decannulation before ICU discharge and that extracting the data from a wide variety of centers without intervening in standard medical practice would increase the strength of our conclusions. Therefore, we aimed to determine the impact of decannulation before ICU discharge on ward survival in nonexperimental conditions by using propensity analysis of the factors associated with decannulation before ICU discharge in data collected from a wide variety of sources for the validation of the Sabadell Score (12).

\section{MATERIALS AND METHODS}

We analyzed data from a prospective, multicenter cohort of 4,132 adult patients who were admitted to 31 ICUs in Spain during a 3-month period beginning March 1, 2008 (the Appendix give a list of centers) (12). The local institutional review board at each participating center approved the study protocol and waived the need for consent. For the purpose of this predefined analysis, we selected only the patients who were tracheostomized during the ICU stay due to prolonged MV.

In a specific Web-based database, we recorded the following variables for each patient admitted to the ICU: 1) on ICU admission, age, sex, diagnosis, predicted risk of death (Acute Physiology and Chronic Health Evaluation II, Simplified Acute Physiology Score 2, or Simplified Acute Physiology Score 3, depending on each ICU's clinical routine), source of admission, and do-not-resuscitate orders; 2) during the ICU stay, 
tracheal intubation, MV, noninvasive MV, vasoactive drugs, parenteral nutrition, blood transfusion, dialysis, tracheostomy, acute renal failure or infection acquired in the ICU, and prognosis assessed by the Sabadell Score (13); 3) after discharge from the ICU, ICU readmission and outcome. In patients readmitted to the ICU, only the first admission was included in the analysis. Briefly, the Sabadell Score classification system delineates four separate groups of patients: score 0, patients with good long-term prognosis; score 1, patients with poor long term prognosis (>6 months) and suitable for ICU readmission without restrictions; score 2, patients with poor prognosis in the short term ( $<6$ months) and with debatable ICU readmission; score 3, patients not expected to survive the hospital stay. The score is fully subjective and integrates the physician's knowledge about the patient's conditions and functional derangement, as well as about the performance of the healthcare facility. The ward team was blind to the Sabadell Score; likewise, ICU physicians evaluating requests for ICU readmission were blind to the Sabadell Score.

Decisions about whether and how to perform tracheostomy in the ICU, as well as whether and how to perform decannulation, remained at the discretion of the attending physicians, who were unaware of the study.

The ward team was also unaware of the study; thus, the care of tracheostomized patients in the ward and decannulation, when feasible, should represent the standard treatment in most of our healthcare system.

Follow-up variables were ICU readmission and 90-day ward outcome, even in patients who were transferred to another acute care hospital.

Statistical Analysis. Categorical variables were expressed as percentages and compared using chi-square tests. Continuous variables were expressed as means and standard deviations and compared by one-way analysis of variance when normally distributed and were expressed as medians and 25\% to 75\% percentiles and compared by KruskalWallis analysis when non-normally distributed. Statistical significance was set at $\mathrm{p}<$ .05 .

The Cox regression estimate-of-survival curve was used for the univariate analysis of hospital mortality. The association of variables with ward mortality was assessed with backward multiple logistic regression.

Since patients were not randomly assigned to decannulation and nondecannulation groups, we developed a propensity score using all the variables predictive of decannulation determined by logistic regression to account for treatment-indication bias and potentially confounding variables. Logistic regression identified the following variables as associated with decannulation: use of vasoactive drugs, ICU admission for non-neurologic disease, and absence of ICU-acquired infection. After testing the balanced distribution of variables across each quintile of the propensity scores, we elaborated a Cox regression estimate-of-survival curve for decannulated and nondecannulated patients, adjusting for quintiles and Sabadell Score as covariates. Analyses were performed with the Stata statistical program (version 10.0; StataCorp, College Station, TX).

\section{RESULTS}

We included 4,132 patients; 1,996 (48\%) needed MV, and 260 (13\%) of these were tracheostomized (Fig. 1). Table 1 shows the characteristics of mechanically ventilated patients grouped as tracheostomized and nontracheostomized patients. As has been commonly reported, tracheostomized patients were sicker and more commonly admitted 
from the ward and for respiratory and neurologic diseases. Tracheostomized patients required more ICU treatments (vasoactive drugs, transfusion, and parenteral nutrition) and had a higher frequency of acute renal failure and ICU-acquired infection as well as a longer stay in the ICU (median stay 28 vs. 5 days, $p=.001$ ). Furthermore, the subjective prognosis at ICU discharge by the Sabadell Score was also worse in survivors needing tracheostomy. Ward length of stay and hospital mortality were also higher in tracheostomized ICU patients than in nontracheostomized MV survivors.

Finally, 59 (23\%) tracheostomized patients died in the ICU, and 201 were discharged to the ward. Among the survivors, 60 (30\%) were decannulated in the ICU and 141 (70\%) were discharged to the ward with a cannula in place.

Table 2 shows the characteristics of tracheostomized patients discharged to the ward according to their decannulation status. In the univariate analysis, the following factors were more common in patients decannulated before ICU discharge: non-neurologic disease (85\% vs. $68 \%, \mathrm{p}=.02)$, vasoactive drugs $(90 \%$ vs. $76 \%, \mathrm{p}=.01)$, parenteral nutrition (55\% vs. 33\%, $\mathrm{p}=.003)$, and acute renal failure (37\% vs. $23 \%, \mathrm{p}=.04)$. These variables were also significantly associated with decannulation in the ICU in the multivariate logistic regression model, making it possible to construct a propensity score for decannulation based on the coefficients of the logistic model. The quintile distribution showed moderate goodness of classification, with a prevalence of decannulation of $51 \%, 32 \%, 25 \%, 24 \%$, and $17 \%$, respectively, with a balanced distribution of the included variables in each quintile (Fig. 2).

Both crude ward mortality (22\% vs. $23 \%, \mathrm{p}=.9)$ and time course of death were similar in decannulated and nondecannulated patients (Fig. 3). Additionally, in the Cox proportional hazard-ratio analysis after adjustment for the propensity score, only the Sabadell Score but not the presence of a tracheostomy can-nula remained significantly associated with ward survival (Table 3). In this analysis, the lack of decannulation in the ICU, with an odds ratio of $0.6(0.3-1.2)(p=.1)$, failed to appear as a risk factor for ward mortality (Fig. 4).

\section{DISCUSSION}

The present study shows that a tracheostomy cannula in place during ward stay after ICU discharge did not increase the risk of mortality in a wide variety of hospitals with close surveillance in the ward. Because clinical characteristics may determine whether patients can be decannulated before ICU discharge, comparing ward survival in decannulated and nondecannulated patients requires strict adjustments for confounding factors. Our tracheostomy rate was low (13\% of the MV patients); in most cases tracheostomy was performed for prolonged ICU stay and in sicker patients. These findings corroborate those of most previous studies $(7,8,14)$, although Clec'h et al (6) found sicker patients in the nontracheostomized group. Nevertheless, even after tracheostomy, a quarter of the patients did not survive the acute illness in the ICU. Tracheostomized survivors have a worse subjective prognosis at ICU discharge than patients who did not need tracheostomy, and accordingly, their ward length of stay and ward mortality were higher.

Our analysis of the differences among tracheostomized patients during the ICU stay revealed that decannulation was more likely in non-neurologic patients after a severe but reversible illness, as evidenced by the higher frequency of acute renal failure and ICU-acquired infections. In a recent survey in the United States, Stelfox et al (15) reported that respiratory therapists and physicians rated the patients' ability to tolerate capping, secretions, cough effectiveness, and level of consciousness as the most 
important factors in the decision to decannulate, with respiratory therapists placing greater emphasis on the ability to tolerate capping and physicians on the level of consciousness. Similar results were recently reported in an international survey in 10 developed countries by the same authors (16).

The prognosis of ICU survivors is an unresolved issue in clinical practice be-cause general severity scores provide very little accuracy in this specific group of patients. The recently validated Sabadell Score (12) depicts four groups of patients with very different outcomes during ward hospitalization after ICU discharge. In the present study, the rate of nondecannulated patients classified as "poor short-term prognosis" or "expected hospital death" by the Sabadell Score was twice that of decannulated patients. Adjusting for this worse prognosis revealed a slight trend indicating that lack of decannulation was actually a protective factor instead of a risk factor for mortality.

The present data contradict those of our previous single-center study (11), which suggested that ICU discharge to the ward with a cannula in place was a risk factor for mortality in the subgroup of patients with a poor prognosis, either in the short term or in the long term. However, the control group in that study was not only tracheostomized and decannulated patients but the whole population of MV patients. Additionally, the lack of a propensity analysis in that study meant that the comparison could not be adjusted to account for potentially confounding factors, such as the likelihood of decannulation.

Some studies may be hindered by suboptimal treatment in the ward in patients with a cannula in place, as suggested by disproportionately early mortality (9). Recently, after systematically reviewing the literature, Garrubba et al (17) suggested that multidisciplinary teams in the ward may improve survival in tracheostomized patients but that the weakness of the studies reviewed precluded strong conclusions. Our ward survival curve shows a progressive decay in survival in both groups, with a slightly faster but statistically nonsignificant decay in decannulated patients, as suggested by a half-time mortality of 8 days in the decannulated group vs. 12 days in the nondecannulated group. Although we have no direct data about the appropriateness of care in the ward, these data suggested that no major flaws in medical care in the ward can account for our main results.

An alternative explanation for the lack of survival advantage in patients decannulated before ICU discharge is that premature decannulation in some patients may expose them to secondary airway protection derangements in the ward. As a result, some attending physicians may consider that some of these patients are not candidates for reintubation. Conversely, they may consider patients discharged with a cannula in place as undergoing a low pacing process requiring frequent reassessment of overall recovery before moving to full decannulation.

Limitations of the Study. Our study is limited by its observational design, which cannot exclude the possibility of our results being confounded by case-mix heterogeneity or secular trends. However, although a prospective, controlled, randomized clinical trial is the optimal means of demonstrating causality, appropriately designed observational studies with the right analytical strategies can provide valuable information on treatment effectiveness. Suarez et al (18) showed that, when analyzed with propensity score methodology, the results obtained in prospective, observational studies might be similar to those obtained in randomized, controlled trials. Furthermore, observational studies maximize external validity, and recent simulation research showed that propensity score methods generally gave treatment effect estimates that were closer to the true treatment effect than logistic regression models in which all confounders were modeled. The distribution of these confounders should be fairly equal between the treatment groups when estimating the effect of treatment on the outcome, as found in the present study. 
Nevertheless, our epidemiologic approach did not include other variables that can also act as confounders about the likelihood of decannulation, such as characteristics of secretions, coughing ability, or level of consciousness $(9,14)$. Our database also lacks data about body mass index, which has been suggested as a risk factor for mortality in the ward in tracheostomized patients (9). Additionally, "presumed good ward performance" is hard to define and lacks homogeneous criteria, so our operational definition relies only on the subjective perception of ICU physicians as to whether they confidently discharge patients to the ward with a tracheostomy cannula in place.

The lack of a common ICU or ward decannulation protocol may have increased the variability of our results. Thus, although our results provide a picture of the day-to-day reality around Spain, we cannot rule out even better outcomes if these fragile patients were surveyed by dedicated teams during the ward stay, as has been recommended (10). The inclusion of the Sabadell Score as a factor in the survival analysis may be disputed because it is not widely used. Nevertheless, a secondary analysis excluding this score confirmed that a tracheostomy cannula in place was not a risk factor for mortality (data not shown) in our patients. Because attending physicians in the ward were unaware of the study, our results could not have been affected by the "Hawthorne effect," i.e., the inadvertent modification of treatments induced by enrollment in a trial.

We conclude that, in our multicenter setting, ICU discharge to the ward without decannulation is not a risk factor.

\section{REFERENCES}

1. Heffner JE: The role of tracheotomy in weaning. Chest 2001; 120:477S481S.

2. MacIntyre N: Discontinuing mechanical ventilatory support. Chest 2007; 132:1049-1056.

3. Nathens AB, Rivara FP, Mack CD, et al: Variations in rates of tracheostomy in the critically ill trauma patient. Crit Care Med 2006; 34:2919-2924.

4. Engoren M, Arslanian-Engoren C, Fenn-Buderer N: Hospital and long-term outcome after tracheostomy for respiratory failure. Chest 2004; 125:220 227.

5. Combes A, Luyt CE, Nieszkowska A, et al: Is tracheostomy associated with better out-comes for patients requiring long-term mechanical ventilation? Crit Care Med 2007; 35:802-807.

6. Clec'h C, Alberti C, Vincent F, et al: Tracheostomy does not improve the outcome of patients requiring prolonged mechanical ventilation: A propensity analysis. Crit Care Med 2007; 35:132-138.

7. Frutos-Vivar F, Esteban A, Apezteguía C, et al: Outcome of mechanically ventilated patients who require a tracheostomy. Crit Care Med 2005; 33:290-298.

8. Gordo F, Núñez A, Calvo E, et al: Intrahospital mortality after discharge from the ICU (hidden mortality) in patients who required mechanical ventilation. Med Clin (Barc) 2003; 121:241-244.

9. Hernandez GH, Fernandez R, Sanchez-Casado MS, et al: Tracheostomy tube in place at intensive care unit discharge is associated with increased ward mortality. Respir Care 2009; 54:1644-1652.

10. Tobin AE, Santamaria JD: An intensivist-led tracheostomy review team is associated with shorter decannulation time and length of stay: A prospective cohort study. Crit Care 2008; 12:R48. 
11. Fernandez R, Bacelar N, Hernandez G, et al: Ward mortality in patients discharged from the ICU with tracheostomy may depend on patient's vulnerability. Intensive Care Med 2008; 34:1878-1882.

12. Fernandez R, Serrano JM, Umaran I, et al: Ward mortality after ICU discharge: A multicenter validation of the Sabadell score. Intensive Care Med 2010; 36:1196-1201.

13. Fernandez R, Baigorri F, Navarro G, et al: A modified McCabe score for stratification of patients after intensive care unit discharge: the Sabadell Score. Crit Care 2006; 10:R179.

14. Kollef MH, Ahrens TS, Shannon W: Clinical predictors and outcomes for patients requiring tracheostomy in the intensive care unit. Crit Care Med 1999; 27:1714-1720.

15. Stelfox HT, Hess DR, Schmidt UH: A North American survey of respiratory therapist and physician tracheostomy decannulation practices. Respir Care 2009; 54:1658-1664.

16. Stelfox HT, Crimi C, Berra L, et al: Determinants of tracheostomy decannulation: An international survey. Crit Care 2008; 12:R26.

17. Garrubba M, Turner T, Grieveson C: Multidisciplinary care for tracheostomy patients: A systematic review. Crit Care 2009; 13:R177.

18. Suarez D, Haro JM, Novick D, et al: Marginal structural models might overcome confounding when analyzing multiple treatment effects in observational studies. JClin Epidemiol 2008; 61:525-530.

\section{APPENDIX}

The Sabadell Score Group members include the following collaborators: Manuel Serrano (Hospital General Universitario Reina Sofia, Cordoba, Spain), Isabel Umaran (Hospital de Cruces, Baracaldo, Spain), Susana Altaba (Hospital General Universitario Castello, Castello, Spain), Andres Carrillo (Hospital General Universitario JM Morales Messeguer, Murcia, Spain), Ma-Jose Lopez-Pueyo (Hospital General Yague, Burgos, Spain), Pedro Rascado (Complexo Hospitalario Universitario de A Coruña, A Coruña, Spain), Begoña Balerdi (Hospital Universitario La Fe, Valencia, Spain), Borja Suberviola (Hospital Universitario Marques de Valdecilla, Santander, Spain), Victoria Lacueva (Hospital de Sagunt, Sagunt, Spain), Rosa-M ${ }^{a}$ Catalan (Corsorci Hospitalari de Vic, Vic, Spain), Lluis Cabre (Hospital de Barcelona SCIAS, Barcelona, Spain), Antonio Santos (Complexo Hospitalario Universitario de Santiago de Compostela, Santiago de Compostela, Spain), Paula Vera (Hospital Sant Joan de Reus, Reus, Spain), $\mathrm{M}^{\mathrm{a}}$-Jose Gutierrez (Hospital San Agustin, Aviles, Spain), Jose-Felipe Solsona (Hospital del Mar, Barcelona, Spain), Jose-Manuel Añon (Hospital Virgen de la Luz, Cuenca, Spain), Enrique Fernandez-Mondejar (Hospital Virgen de las Nieves, Granada, Spain), Ramon Fernandez-Cid (Hospital Mateu Orfila, Menorca, Spain), Emilio Curiel (Hospital Universitario Carlos Haya (Hospital Civil), Malaga, Spain), Vicente GomezTello (Hospital Moncloa, Madrid, Spain). 


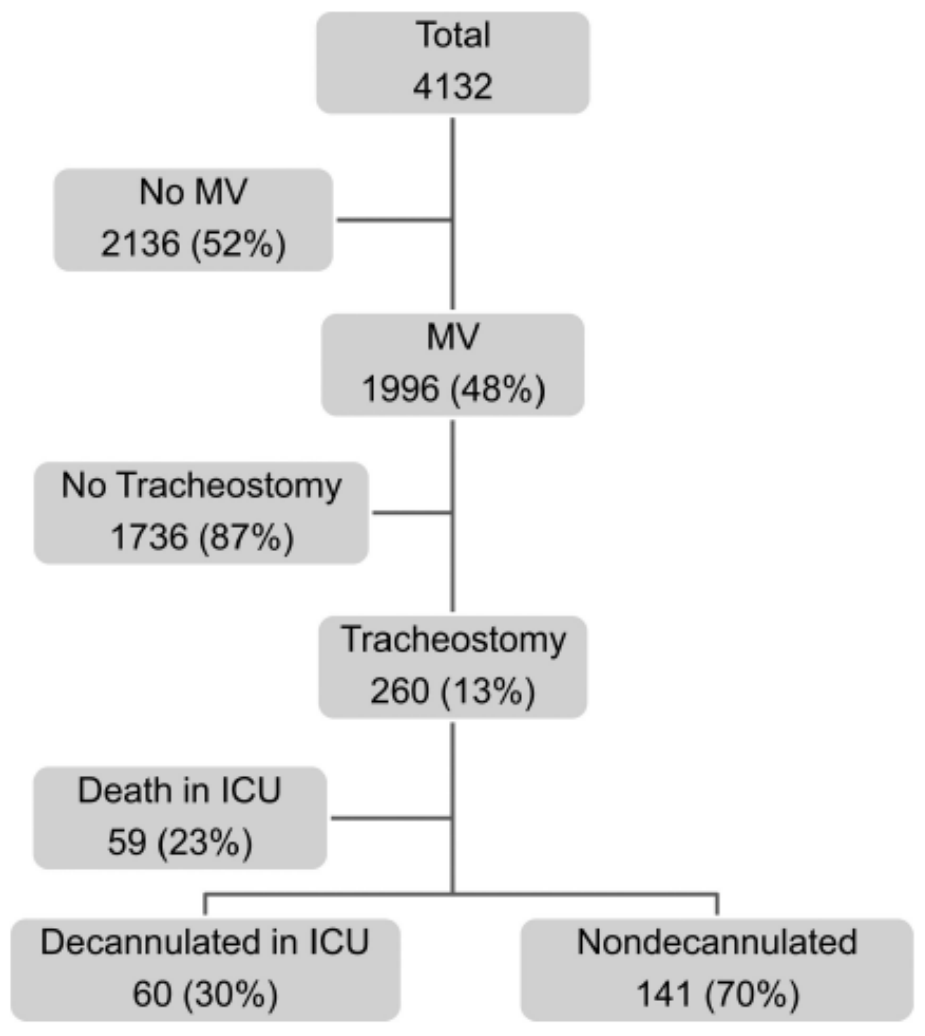

Figure 1. Flowchart of the study. $M V$, mechanical ventilation; $I C U$, intensive care unit.

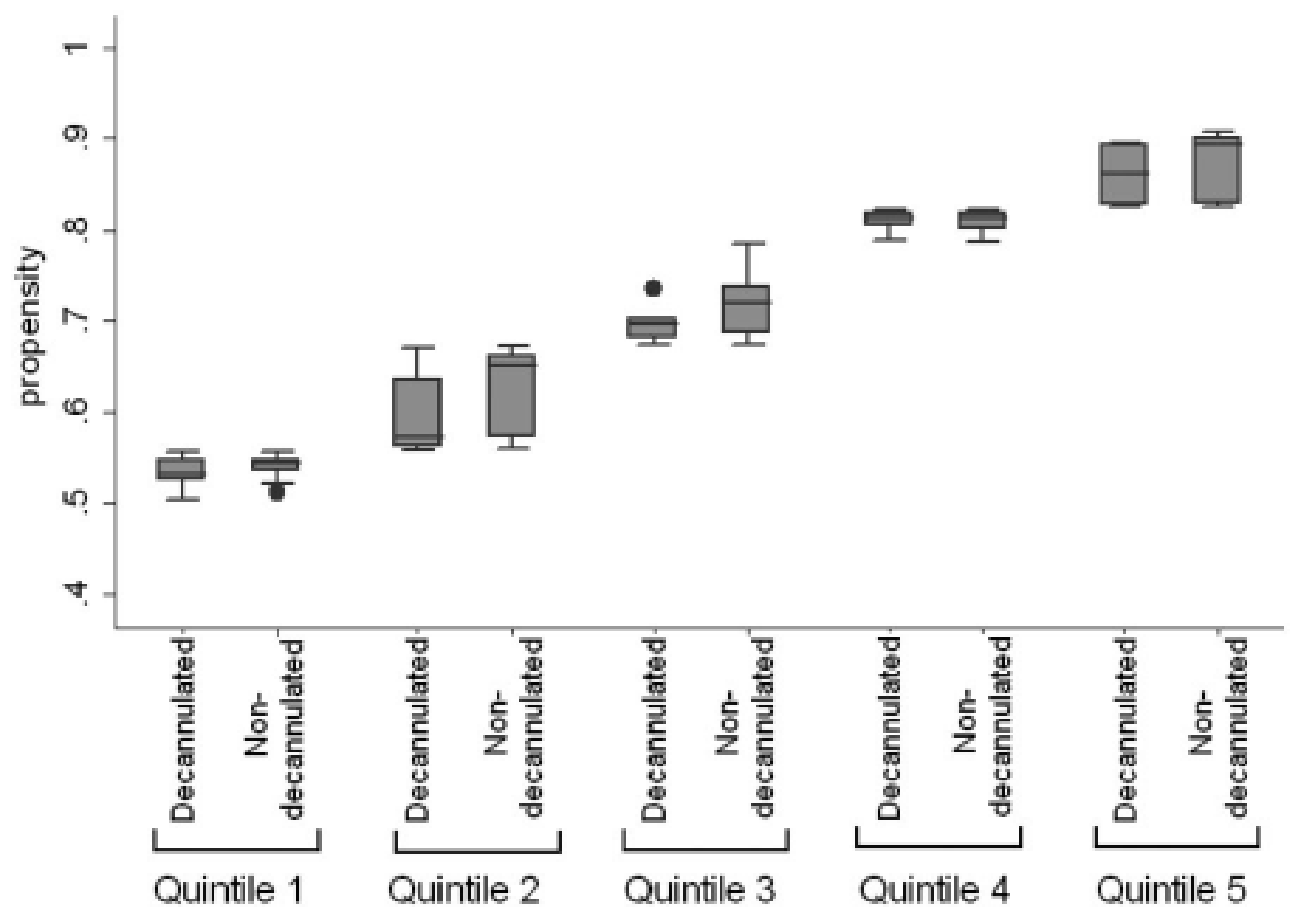

Figure 2. Box-plot chart showing balanced propensity scores for decannulated and nondecannulated patients in each quintile. Dots indicate outliers. 


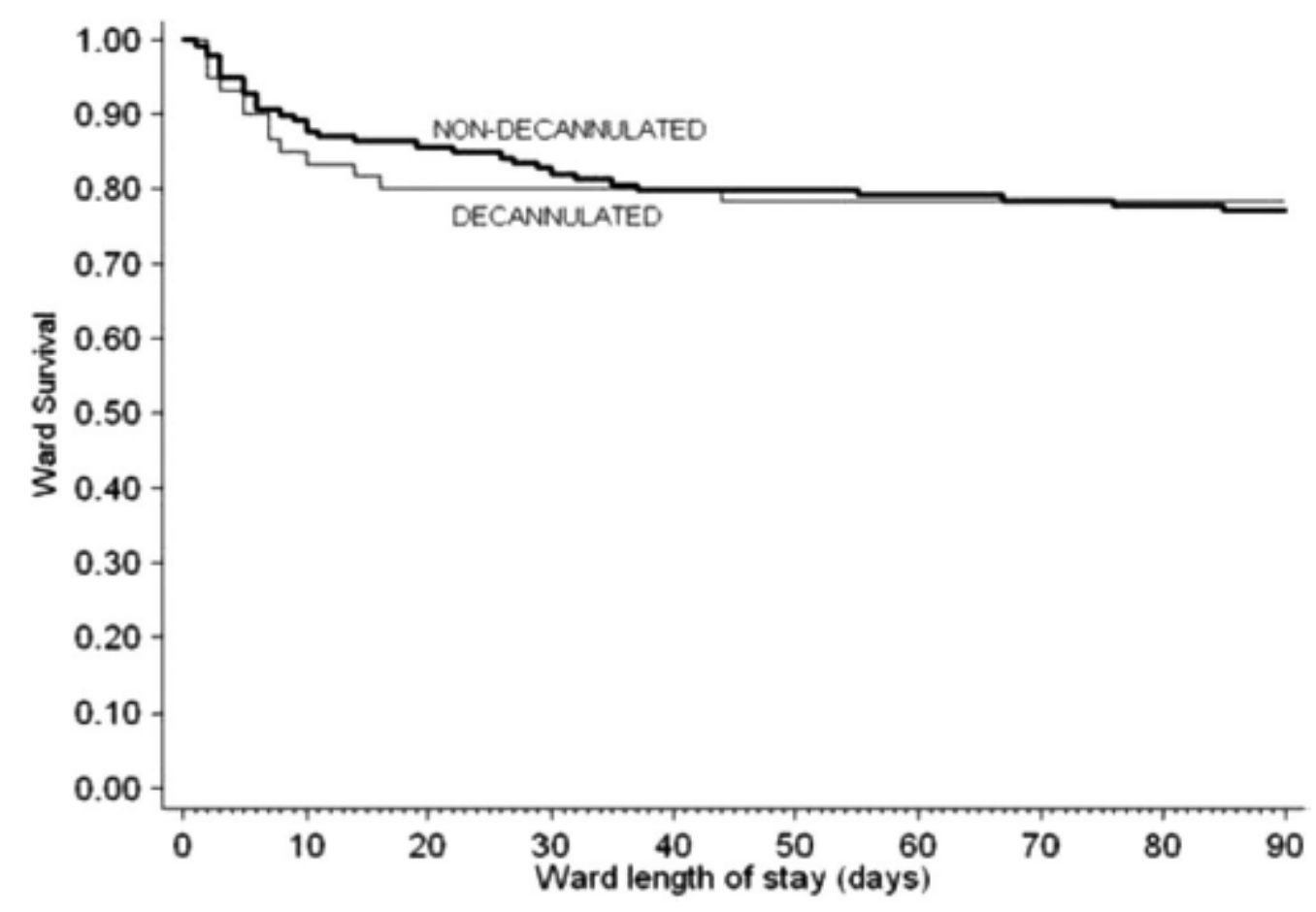

Figure 3. Survival curve for decannulated and nondecannulated patients in the ward ( $p$ is not significant).

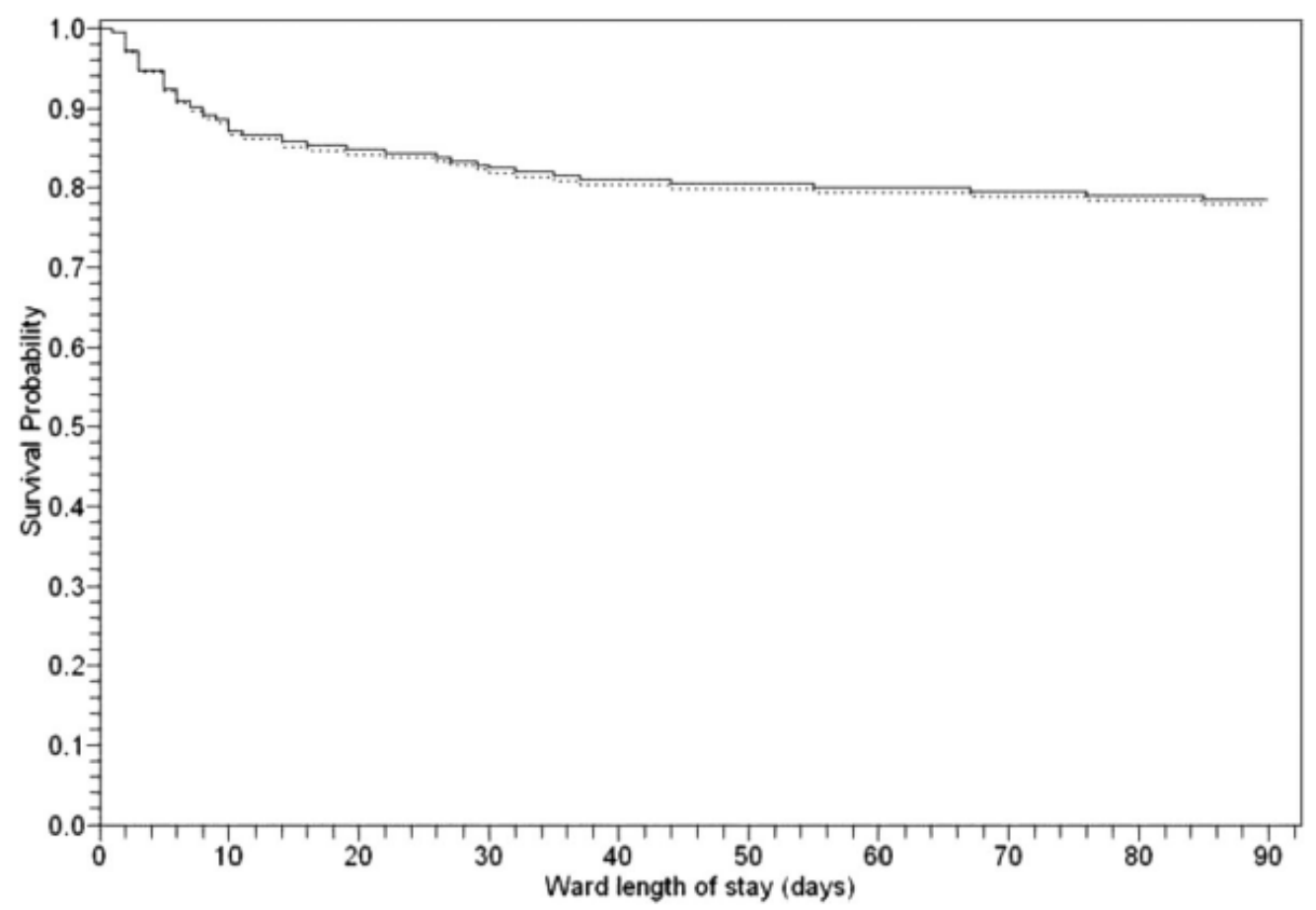

Figure 4. Cox proportional hazard function for ward survival of decannulated (dotted line) and nondecannulated (solid line) patients after adjustment for quintiles of the propensity score and Sabadell Score ( $p$ is not significant). 


\begin{tabular}{|c|c|c|c|}
\hline Characteristic & $\begin{array}{c}\text { Tracheostomy } \\
(n=260)\end{array}$ & \begin{tabular}{|c|} 
No Tracheostomy \\
$(n=1,736)$
\end{tabular} & $p$ \\
\hline Age, yrs & $62.3 \pm 16.0$ & $61.5 \pm 16.2$ & .5 \\
\hline Male sex & $180(69 \%)$ & $1,128(65 \%)$ & .1 \\
\hline Predicted risk of death, \% & $37 \pm 23$ & $31 \pm 26$ & .001 \\
\hline Source of admission & & & .001 \\
\hline Operating room & $46(18 \%)$ & $670(39 \%)$ & \\
\hline Emergency room & $101(39 \%)$ & $606(35 \%)$ & \\
\hline Ward & $83(32 \%)$ & $343(20 \%)$ & \\
\hline Other & $30(11 \%)$ & $117(7 \%)$ & \\
\hline Diagnostic group & & & .04 \\
\hline Postsurgical & $41(16 \%)$ & $472(28 \%)$ & \\
\hline Cardiovascular & $33(13 \%)$ & $380(22 \%)$ & \\
\hline Respiratory & $59(23 \%)$ & $300(17 \%)$ & \\
\hline Neurologic & $64(25 \%)$ & $273(16 \%)$ & \\
\hline Trauma & $38(15 \%)$ & $115(7 \%)$ & \\
\hline Coronary & $3(1 \%)$ & $44(2 \%)$ & \\
\hline Other & $22(8 \%)$ & $152(9 \%)$ & \\
\hline Do-not-resuscitate orders on admission & $18(7 \%)$ & $113(6 \%)$ & .4 \\
\hline Vasoactive drugs & $218(84 \%)$ & $1082(62 \%)$ & .001 \\
\hline Transfusion & $170(65 \%)$ & $665(38 \%)$ & .001 \\
\hline Acute renal failure & $95(36 \%)$ & $483(28 \%)$ & .003 \\
\hline Parenteral nutrition & $119(46 \%)$ & $389(22 \%)$ & .001 \\
\hline Noninvasive ventilation & $55(21 \%)$ & $233(13 \%)$ & .001 \\
\hline ICU-acquired infection & $183(70 \%)$ & $216(12 \%)$ & .001 \\
\hline ICU length of stay, days & $28.5(17-41)$ & $5(3-10)$ & .001 \\
\hline ICU mortality & $59(23 \%)$ & $418(24 \%)$ & .9 \\
\hline Prognosis at ICU discharge & & & .001 \\
\hline Good prognosis & $49(19 \%)$ & $870(50 \%)$ & \\
\hline Poor long-term prognosis & $67(26 \%)$ & $296(17 \%)$ & \\
\hline Poor short-term prognosis & $52(20 \%)$ & $114(7 \%)$ & \\
\hline Expected to die in hospital & $33(13 \%)$ & $38(2 \%)$ & \\
\hline Ward length of stay, days & $12.5(1-34)$ & $8(1-15)$ & .001 \\
\hline Hospital mortality & $104(40 \%)$ & $509(29 \%)$ & .001 \\
\hline
\end{tabular}

ICU, intensive care unit. 
Table 2. Clinical variables of tracheostomized patients classified according to whether they were decannulated in the intensive care unit

\begin{tabular}{|c|c|c|c|}
\hline Characteristic & $\begin{array}{c}\text { Decannulated } \\
(\mathrm{n}=60)\end{array}$ & \begin{tabular}{|c|}
$\begin{array}{c}\text { Nondecannulated } \\
(\mathrm{n}=141)\end{array}$ \\
\end{tabular} & $p$ \\
\hline Age, yrs & $61.3 \pm 17.0$ & $60.2 \pm 16.9$ & .7 \\
\hline Male sex & $44(73 \%)$ & $94(67 \%)$ & .2 \\
\hline Predicted risk of death, \% & $35 \pm 23.9$ & $33 \pm 21.1$ & .5 \\
\hline Source of admission & & & .4 \\
\hline Operating room & $13(22 \%)$ & $20(14 \%)$ & \\
\hline Emergency room & $22(37 \%)$ & $63(45 \%)$ & \\
\hline Ward & $19(32 \%)$ & $39(28 \%)$ & \\
\hline Other & $6(10 \%)$ & $19(13 \%)$ & \\
\hline Diagnostic group & & & .02 \\
\hline Postsurgical & $11(18 \%)$ & $19(13 \%)$ & \\
\hline Cardiovascular & $10(17 \%)$ & $11(8 \%)$ & \\
\hline Respiratory & $15(25 \%)$ & $29(21 \%)$ & \\
\hline Neurologic & $9(15 \%)$ & $45(32 \%)$ & \\
\hline Trauma & $8(13 \%)$ & $28(20 \%)$ & \\
\hline Coronary & $2(3 \%)$ & $0(0 \%)$ & \\
\hline Other & $5(8 \%)$ & $9(6 \%)$ & \\
\hline Do-not-resuscitate orders on admission & $4(7 \%)$ & $6(4 \%)$ & .3 \\
\hline Vasoactive drugs & $54(90 \%)$ & $107(76 \%)$ & .01 \\
\hline Transfusion & $42(70 \%)$ & $84(60 \%)$ & .1 \\
\hline Acute renal failure & $22(37 \%)$ & $33(23 \%)$ & .04 \\
\hline Parenteral nutrition & $33(55 \%)$ & $47(33 \%)$ & .003 \\
\hline Noninvasive ventilation & $12(20 \%)$ & $27(19 \%)$ & .5 \\
\hline ICU-acquired infection & $38(63 \%)$ & $100(71 \%)$ & .2 \\
\hline ICU length of stay, days & $29.5(18-41.5)$ & $27(16-40)$ & .3 \\
\hline Prognosis at ICU discharge & & & .002 \\
\hline Good prognosis & $24(40 \%)$ & $25(18 \%)$ & \\
\hline Poor long-term prognosis & $21(35 \%)$ & $46(33 \%)$ & \\
\hline Poor short-term prognosis & $9(15 \%)$ & $43(30 \%)$ & \\
\hline Expected to die in hospital & $6(10 \%)$ & $27(19 \%)$ & \\
\hline ICU readmission & $6(11 \%)$ & $7(5 \%)$ & .1 \\
\hline Ward length of stay, days & $14(7-32)$ & $25(9-49)$ & .01 \\
\hline Hospital mortality & $13(22 \%)$ & $32(23 \%)$ & .5 \\
\hline
\end{tabular}

ICU, intensive care unit. 
Table 3. Cox proportional hazards analysis for ward mortality.

\begin{tabular}{|l|c|c|}
\hline Term & $\begin{array}{c}\text { Hazard Ratio (95\% } \\
\text { Confidence Interval) }\end{array}$ & $\boldsymbol{p}$ \\
\hline Nondecannulation & $0.6(0.3-1.2)$ & .1 \\
\hline Sabadell Score (1 vs. 0) & $6.9(0.9-56.0)$ & .07 \\
\hline Sabadell Score (2 vs. 0) & $25.4(3.3-197.1)$ & .002 \\
\hline Sabadell Score (3 vs. 0) & $59.4(7.8-453.7)$ & .001 \\
\hline Quintile (2 vs. 1) & $0.5(0.2-1.4)$ & .2 \\
\hline Quintile (3 vs. 1) & $0.3(0.1-0.9)$ & .05 \\
\hline Quintile (4 vs. 1) & $0.8(0.3-2.0)$ & .5 \\
\hline Quintile (5 vs. 1) & $0.7(0.3-1.9)$ & .6 \\
\hline
\end{tabular}

